\title{
WEAK CONDITIONS FOR GENERATION OF COSINE FAMILIES IN LINEAR TOPOLOGICAL SPACES
}

\author{
MICHIAKI WATANABE
}

(Communicated by Walter Littman)

\begin{abstract}
Let $A$ be a closed linear operator in a Banach space $X$. Weak

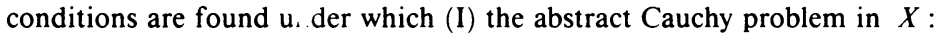

$$
u^{\prime \prime}(t)=A u(t), \quad t \in R ; \quad u(0)=u_{0}, \quad u^{\prime}(0)=u_{1}
$$

has a unique solution for each $u_{0}$ an $\mathrm{d} u_{1}$ given in a dense subset $Y$ of $X$, and (II) the set $Y$ becomes a linear topological space where $\left.A\right|_{Y}$ generates a continuous cosine family.

The conditions are satisfied for example by the generator of a strongly continuous or holomorphic semigroup in $X$.
\end{abstract}

\section{INTRODUCTION}

Let $A$ be a closed linear operator in a Banach space $X$ with norm $\|\cdot\|$, and consider the Cauchy problem for the second-order evolution equation in $X$ :

$$
\begin{gathered}
u^{\prime \prime}(t)=A u(t), \quad t \in R \\
u(0)=u_{0} \quad \text { and } \quad u^{\prime}(0)=u_{1},
\end{gathered}
$$

where ${ }^{\prime}=d / d t$. Concerning the operator-theoretical treatment, we have the condition due to Sova [9] and Da Prato and Giusti [3] (see Fattorini [5] and also [10]):

(A) The domain $D(A)$ of $A$ is dense in $X$. There are positive constants $M$ and $\omega$ such that the subset $\left\{z^{2}: \operatorname{Re} z>\omega\right\}$ of the complex plane $C$ is included in the resolvent set $\rho(A)$ of $A$, and

$$
\left\|(1 / n !)(d / d z)^{n} z\left(z^{2}-A\right)^{-1} x\right\| \leq M(\operatorname{Re} z-\omega)^{-n-1}\|x\|
$$

holds for $x \in X, \operatorname{Re} z>\omega$ and $n=0,1, \ldots$.

This means the existence of a strongly continuous cosine family $\{C(t): t \in$ $R\}$ in $X$ : For $x \in X \quad t \rightarrow C(t) x$ is continuous;

$$
\begin{aligned}
C\left(t+t^{\prime}\right)+C\left(t-t^{\prime}\right) & =2 C(t) C\left(t^{\prime}\right) \text { for } t, t^{\prime} \in R, \\
C(0) & =I ;
\end{aligned}
$$

Received by the editors February 20, 1988.

1980 Mathematics Subject Classification (1985 Revision). Primary 35L30, 34G10, 47D99.

Key words and phrases. Evolution equation of second order, cosine family, abstract Gevrey space.

(C) 1989 American Mathematical Society $0002-9939 / 89 \$ 1.00+\$ .25$ per page 
and the generator $A$ is defined by $A u=\left.(d / d t)^{2} C(t) u\right|_{t=0}$ with $D(A)=\{u \in$ $X: t \rightarrow C(t) u$ is of class $\left.C^{2}\right\}$. But (3) seems to be rather difficult to verify directly for a given differential operator (see Sova [9] and also [10]). This is very different from the case of the Hille-Yosida-Phillips theorem on semigroup generation. Yosida [12] solved (1)-(2) for a concrete wave equation by constructing a strongly continuous group in terms of the matrix operator $\left(\begin{array}{ll}0 & I \\ A & 0\end{array}\right)$. Kisyński [8] pointed out that $(\mathrm{A})$ is equivalent to the condition:

(B) There exists a Banach space $V$ including $D(A)$ and included continuously in $X$ such that the operator $\left(\begin{array}{ll}0 & I \\ A & 0\end{array}\right)$ with domain $D(A) \times V$ generates a strongly continuous group in the Banach space $V \times X$.

Suggested by

$$
\left(\begin{array}{cc}
0 & I \\
A^{1 / 2} A^{1 / 2} & 0
\end{array}\right) \text { or }\left(\begin{array}{cc}
0 & A^{1 / 2} \\
A^{1 / 2} & 0
\end{array}\right)
$$

Fattorini [4, 5], Goldstein [6], and recently Yagi [11] solved (1)-(2) through the group generated by $A^{1 / 2}=i(-A)^{1 / 2}, i=(-1)^{1 / 2}$ (in the exact or a formal sense) for a class of operators. However it is still highly desirable to find out a convenient criterion for (3) or a condition weaker than (A) or (B).

The purpose of this paper is to deal with the problem (1)-(2) under the following condition on $A$ :

(C) $A$ is a closed linear operator with domain dense in $X$. There are constants $M>0,0<\theta<\pi$ and $k \geq-1$ such that the sector $|\arg z| \leq \theta$ in $C$ is included in $\rho(A)$ and

$$
\left\|(z-A)^{-1} x\right\| \leq M|z|^{k}\|x\|
$$

holds for $x \in X$ and $|\arg z| \leq \theta$, where we assume, by replacement by $A$ of $A-r I$ for $r>0$ if necessary, that a neighborhood of 0 is included in $\rho(A)$.

The condition (C) is weaker than (A). In fact, under (A) a holomorphic semigroup in $X$ is generated by $A$; under (C) even a strongly continuous semigroup in $X$ is not necessarily generated by $A$. In other words, any operator which generates a strongly continuous or holomorphic semigroup in $X$ satisfies (C). As is well known, if $A$ satisfies (C) with $k=-1$, fractional powers of $-A$ are defined by the Balakrishnan-Kato-Yosida approach. Guzman [7, Theorems 1.1 and 1.2] characterized the generator of a holomorphic semigroup in $X$ having among other things a slow growth like $O\left(t^{h}\right),-1<h<0$ as $t \downarrow 0$ in terms of a fractional power of $-A$ under $(\mathrm{C}),-1<k<0$. It is therefore needless to look for examples of concrete differential operators illustrating $(C)$.

The Cauchy problem (1)-(2) in $X$ is to find a function $u(t)$ of $t \in R$ with values in $X$ such that $u(t)$ belongs to $D(A)$ for $t \in R$, and $t \rightarrow u(t)$ is of class $C^{2}$ and satisfies (1) and (2). The main results of this paper are as follows.

Theorem 1. If an operator $A$ satisfies the condition (C), then there is a dense subset $Y$ of $X$ such that the problem (1)-(2) in $X$ has a unique solution for each given $u_{0}$ and $u_{1}$ in $Y$. 
Theorem 2. Under (C), the set $Y$ becomes a linear topological space and the restriction $\left.A\right|_{Y}$ of $A$ in $Y$ generates a continuous cosine family in $Y$.

Moreover, $Y$ is identified as an abstract Gevrey space associated with $A$ of order $a$ with $2-2 \theta / \pi<a<2$.

A problem of similar nature was discussed by Beals [1]: He dealt with the equation of first order $u^{\prime}(t)=A u(t), t \geq 0$ in $X$ under a condition on $A$ somewhat stronger than ours, and consequently solved "weakly hyperbolic" partial differential equations (see [2]). The idea of using nice linear topological spaces is also available to our problem (1)-(2) for the equation in $X$ of "hyperbolic" type.

The operator valued function of $t \in R$ and $s>0$ :

$$
C(t, s)=(2 \pi i)^{-1} \int_{Q} e^{-s(-z)^{b}} \cos \left(t(-z)^{1 / 2}\right)(z-A)^{-1} d z
$$

defined with a suitable path $Q$ and $b>\frac{1}{2}$ plays an essential role throughout. This integral was once used with $b=1$ by Da Prato and Giusti [3, p. 359] to construct a strongly continuous cosine family in $X$ under the condition (A). The main task in this paper is, roughly speaking, to show that for each $u_{0} \in Y$, $C(t, s) C(0, s)^{-1} u_{0}$ is a unique solution of (1)-(2) with $u_{1}=0$ and next that for each $t \in R, C(t, s) C(0, s)^{-1}$ becomes a continuous operator $C(t): Y \rightarrow Y$ independently of $s>0$ and $\{C(t): t \in R\}$ satisfies (4).

\section{The Cauchy problem in $X$}

We shall begin this section with a discussion of the function $C(t, s)$ under the condition $(\mathrm{C})$ and then give the proof of Theorem 1 .

Let $b$ satisfy $\frac{1}{2}<b<(2-2 \theta / \pi)^{-1}$, and let $(-z)^{b}$ be the branch of the power function which is holomorphic off the nonnegative real axis and positive for $z<0$. Let $S$ be the sector $\{s \in C:|\arg s|<d\}$, where $d$ is a constant with $0<d<\pi / 2-b(\pi-\theta)$. Consider the function of $t \in R$ and $s \in S$

$$
C(t, s)=(2 \pi i)^{-1} \int_{Q} e^{-s(-z)^{b}} C(t ; z)(z-A)^{-1} d z,
$$

where $C(t ; z)=\sum_{m=0}^{\infty} t^{2 m} z^{m} /(2 m)$ ! and $Q$ is an integral path which runs in $\rho(A)$ from $\infty e^{i(2 \pi-\theta)}$ to $\infty e^{i \theta}$ avoiding the nonnegative real axis, for instance

$$
\begin{gathered}
\{z: \arg z=2 \pi-\theta,|z|>p\} \cup\{z:|z|=p, \theta \leq \arg z \leq 2 \pi-\theta\} \\
\cup\{z: \arg z=\theta,|z|>p\}
\end{gathered}
$$

with a sufficiently small $p>0$.

Since $\cos (\arg s+b \arg (-z))$ is not smaller than the constant $c=$ $\cos (d+b(\pi-\theta))$ with $0<c<1$ for any $s \in S$ and $z \in Q$ with large $|z|$, we have the estimate

$$
\left|e^{-s(-z)^{b}}\right| \leq e^{-c|s||z|^{b}}
$$


Thus using the estimate $|C(t ; z)| \leq e^{|t||z|^{1 / 2}}$ together with (5), we obtain that the integral (6) converges uniformly on every bounded $t$-subinterval of $R$ for each fixed $s \in S$. Hence $t \rightarrow C(t, s)$ is continuous from $R$ to the bounded operators in $X$.

Next consider the associated function $S(t, s)$ defined by

$$
S(t, s)=\int_{0}^{t} C(r, s) d r
$$

for $t \in R$ and $s \in S$. Clearly we have

$$
S(t, s)=(2 \pi i)^{-1} \int_{Q} e^{-s(-z)^{b}} S(t ; z)(z-A)^{-1} d z,
$$

where $S(t ; z)=\sum_{m=0}^{\infty} t^{2 m+1} z^{m} /(2 m+1) !$.

The following lemma is the most important step of this paper (cf. Da Prato and Giusti [3, Proposition 2]).

Lemma 1. For all $t, t^{\prime} \in R$ and $s, s^{\prime} \in S$,

$$
C\left(t+t^{\prime}, s+s^{\prime}\right)+C\left(t-t^{\prime}, s+s^{\prime}\right)=2 C(t, s) C\left(t^{\prime}, s^{\prime}\right) \text {. }
$$

Proof. Let $Q^{\prime}$ be a path similar to $Q$, defined for $0<p^{\prime}<p$ and $(1-$ $1 / 2 b) \pi+d / b<\theta^{\prime}<\theta$. Clearly an estimate similar to (7) also holds true for $s \in S$ and $w \in Q^{\prime}$ with large $|w|$, and $Q$ may be replaced by $Q^{\prime}$ in (6).

Using the resolvent equation $(z-A)^{-1}-(w-A)^{-1}=(w-z)(z-A)^{-1}(w-$ $A)^{-1}$, we have

$$
\begin{aligned}
C(t, s) C\left(t^{\prime}, s^{\prime}\right)= & (2 \pi i)^{-2} \int_{Q} \int_{Q^{\prime}} e^{-s^{\prime}(-w)^{\prime}} C\left(t^{\prime} ; w\right)(w-z)^{-1} d w \\
& \cdot e^{-s(-z)^{b}} C(t ; z)(z-A)^{-1} d z \\
& +(2 \pi i)^{-2} \int_{Q^{\prime}} \int_{Q} e^{-s(-z)^{b}} C(t ; z)(z-w)^{-1} d z \\
& \cdot e^{-s^{\prime}(-w)^{\prime}} C\left(t^{\prime} ; w\right)(w-A)^{-1} d w .
\end{aligned}
$$

The second term vanishes and the first equals

$$
(2 \pi i)^{-1} \int_{Q} e^{-\left(s+s^{\prime}\right)(-z)^{\prime \prime}} C(t ; z) C\left(t^{\prime} ; z\right)(z-A)^{-1} d z .
$$

which gives (9) since

$$
2 C(t ; z) C\left(t^{\prime} ; z\right)=C\left(t+t^{\prime} ; z\right)+C\left(t-t^{\prime} ; z\right) \text {. Q.E.D. }
$$

Lemma 2. (i) For each $t \in R, C(t, s)$ and $S(t, s)$ are holomorphic functions of $s \in S$;

(ii) $C(t, s) X$ and $S(t, s) X$ are included in $D\left(A^{n}\right), n=1,2, \ldots$, and, in particular, for $t \in R$ and $s \in S$

$$
\left\{\begin{array}{c}
(\partial / \partial t) C(t, s)=A S(t, s) \\
(\partial / \partial t)^{2} C(t, s)=A C(t, s)
\end{array}\right.
$$


Proof. The first assertion (i) is evident from (6) and (8) together with (7). Next differentiation in $t$ of $(6)$ is possible. In fact, the integral over $Q$ of $e^{-s(-z)^{b}} S(t ; z) z(z-A)^{-1}$ converges uniformly on every bounded $t$-subinterval of $R$ for each $s \in S$. Since $A$ is closed and $z(z-A)^{-1}=I+$ $A(z-A)^{-1}$, the first equality of (10) is true. The remaining part of (ii) is clear. Q.E.D.

We shall deal with the function $U(s)=C(0, s)$ of $s \in S$. Taking $t=t^{\prime}=0$ in (9), we obtain

$$
U\left(s+s^{\prime}\right)=U(s) U\left(s^{\prime}\right) \text { for } s, s^{\prime} \in S
$$

The following lemma was established by Beals [1, Lemmas 1 and 3] under a slightly stronger condition. But his method of proof is still available to our operator $A$ satisfying satisfying (C).

Lemma 3. (i) For integer $N$ with $k \leq N<k+1$ and $u \in D\left(A^{N+2}\right), U(s) u \rightarrow u$ in $X$ as $s \rightarrow 0$ in $S$.

(ii) For each $s \in S, U(s)$ is one-one.

We can show here that our condition (C) on $A$ guarantees uniqueness of the solution of (1)-(2) in $X$.

Proposition 4. Under the condition (C), the Cauchy problem (1)-(2) in X has at most one solution.

Proof. Let $u(t)$ be a solution of (1)-(2) with $u_{0}=u_{1}=0$. For $t \geq 0$ and $s>0$, consider the function

$$
f(r)=C(t-r, s) u^{\prime}(r)+A S(t-r, s) u(r), \quad 0 \leq r \leq t \quad(\text { cf. [4, p. 91] }) .
$$

Noting that $A$ and $S(t, s)$ commute and using (10), we obtain $f^{\prime}(r)=0$, which together with $f(0)=0$ implies $f(r)=0$ for $0 \leq r \leq t$. In particular, $U(s) u^{\prime}(t)=f(t)=0$. Lemma 3(ii) implies $u^{\prime}(t)=0$, and hence $u(t)=0$ for $t \geq 0$. Same reasoning for $t<0$. Q.E.D.

Proof of Theorem 1. From (11) we obtain

$$
U\left(s_{1}\right) X \supset U\left(s_{2}\right) X \quad \text { if } 0<s_{1}<s_{2} .
$$

Set $Y=\bigcup_{s>0} U(s) X$. Since $D(A)$ is dense in $X, D\left(A^{2}\right)=A^{-1} D(A)$ is dense in $D(A)$, and inductively $D\left(A^{n+1}\right)$ is dense in $D\left(A^{n}\right)$, hence in $X$. Thus $D\left(A^{N+2}\right)$ is dense in $X$ and, by Lemma 3(i), $Y$ is dense in $X$.

Let $u_{0}$ and $u_{1}$ belong to $Y$. Then, by (12), $u_{0}$ and $u_{1}$ belong to $U(s) X$ for some $s>0$. Setting

$$
u(t)=C(t, s) U(s)^{-1} u_{0}+S(t, s) U(s)^{-1} u_{1}
$$

for $t \in R$ and using (10) and Proposition 4, we see that $u(t)$ is the desired solution of the problem (1)-(2) in $X$. Q.E.D. 


\section{The COSINE FAMILY IN $Y$}

In this section we shall show under the condition (C) that the function $C(t, s) U(s)^{-1}$ defines a cosine family in $Y$ equipped with a suitable topology, and then give the proof of Theorem 2.

Since $U(s)^{-1}$ is closed for each $s>0, U(s) X$ is a Banach space with the graph norm. The inclusion (12) implies that $Y$ becomes a linear topological space when equipped with the inductive limit topology.

Lemma 5. For each $t \in R$ and $s_{1}, s_{2}>0$

$$
U\left(s_{1}\right) C\left(t, s_{2}\right)=C\left(t, s_{2}\right) U\left(s_{1}\right)=C\left(t, s_{1}+s_{2}\right) .
$$

If $0<s_{1}<s_{2}$, then $C\left(t, s_{2}\right) X \subset U\left(s_{1}\right) X$ and

$$
U\left(s_{1}\right)^{-1} C\left(t, s_{2}\right)=C\left(t, s_{2}-s_{1}\right) .
$$

Proof. Since $C(-t, s)=C(t, s)$, (9) with $t=0$ or $t^{\prime}=0$ gives (13). The second assertion is a simple consequence of the first. Q.E.D.

Lemma 6. For $t \in R$ and $u \in U(s) X$ with $s>0$ the following hold:

$$
\begin{gathered}
C(t, w) U(w)^{-1} u=C(t, s) U(s)^{-1} u \text { for } 0<w<s ; \\
C(t, s) U(s)^{-1} u \text { belongs to } U(w) X \text { for } 0<w<s .
\end{gathered}
$$

Proof. From (11) and (13) we obtain that

$$
\begin{aligned}
C(t, s) U(s)^{-1} u & =C(t, w) U(s-w) U(s)^{-1} u \\
& =C(t, w) U(w)^{-1} U(w) U(s-w) U(s)^{-1} u \\
& =C(t, w) U(w)^{-1} u
\end{aligned}
$$

for $0<w<s$. From (14) we see that

$$
\begin{aligned}
C(t, s) U(s)^{-1} & =U(w) U(w)^{-1} C(t, s) U(s)^{-1} u \\
& =U(w) C(t, s-w) U(s)^{-1} u
\end{aligned}
$$

for $0<w<s$, which implies the second assertion. Q.E.D.

Lemma 6 shows that if $0<w<s$, then $C(t, s) U(s)^{-1}$ maps $U(s) X$ into $U(w) X$, and $C(t, s) U(s)^{-1}$ and $C(t, w) U(w)^{-1}$ coincide on $U(s) X$. Thus for each $t \in R$, there is a unique continuous operator $C(t): Y \rightarrow Y$ such that

$$
C(t) u=C(t, s) U(s)^{-1} u
$$

for all $s>0$ and $u \in U(s) X$.

Lemma 7. The family $\{C(t): t \in R\}$ satisfies (4).

Proof. For every $s>0$ and $u \in U(s) X$,

$$
C(0) u=C(0, s) U(s)^{-1} u=u
$$


and therefore $C(0)=I$.

Next we have, by (9) and (14), that

$$
\begin{aligned}
\{C(t & \left.\left.+t^{\prime}\right)+C\left(t-t^{\prime}\right)\right\} u=C\left(t+t^{\prime}, s\right) U(s)^{-1} u+C\left(t-t^{\prime}, s\right) U(s)^{-1} u \\
& =2 C(t, s / 2) C\left(t^{\prime}, s / 2\right) U(s)^{-1} u \\
& =2 C(t, s / 2) U(s / 2)^{-1} C\left(t^{\prime}, s\right) U(s)^{-1} u \\
& =2 C(t) C\left(t^{\prime}\right) u \text { Q.E.D. }
\end{aligned}
$$

Recalling $S(t, s)=\int_{0}^{t} C(r, s) d r$, we find that Lemma 6 also holds true with $C(t, s)$

replaced by $S(t, s)$ and therefore that the the associated sine family $\{S(t): t \in$ $R\}$ can be defined similarly by

$$
S(t) u=S(t, s) U(s)^{-1} u
$$

for all $s>0$ and $u \in U(s) X$.

Proof of Theorem 2. We see first from the equality

$$
A U(s)=U(w) A U(s-w)
$$

that $A$ maps $U(s) X$ into $U(w) X$ for $0<w<s$, and the restriction $\left.A\right|_{Y}$ of $A$ in $Y$ is continuous.

Recalling (10), we obtain that $t \rightarrow C(t, s)$ is continuous and of class $C^{2}$ from $R$ to the bounded operators in $X$. Thus $t \rightarrow C(t)$ is continuous and of class $C^{2}$ to the bounded operators mapping $U(s) X$ into $U(w) X$ for $0<w<$ $s$, and

$$
\left\{\begin{aligned}
(d / d t) C(t) & =\left.A\right|_{Y} S(t), \\
(d / d t)^{2} C(t) & =\left.A\right|_{Y} C(t)
\end{aligned}\right.
$$

hold for $t \in R$, in particular, $\left.A\right|_{Y}=\left.(d / d t)^{2} C(t)\right|_{t=0}$.

Finally we shall compare $Y$ with an abstract Gevrey space of order $a=1 / b$ with $1 / 2<b<(2-2 \theta / \pi)^{-1}$. Let $Y(h)$ be for each $h>0$ the totality of $u \in X$ such that

$$
\left\{\begin{array}{l}
u \in D\left(A^{n}\right) \quad \text { for } n=1,2, \ldots ; \\
|u|_{h}=\sup _{n \geq 1} h^{a n} \Gamma(a n)^{-1}\left\|A^{n} u\right\|<\infty .
\end{array}\right.
$$

Clearly $Y(h)$ becomes a Banach space with norm $|\cdot|_{h}$ and satisfies

$$
Y\left(h_{1}\right) \supset Y\left(h_{2}\right) \text { if } 0<h_{1}<h_{2},
$$

and hence $\bigcup_{h>0} Y(h)$ becomes a Gevrey space of order $a$ when equipped with the usual inductive limit topology.

According to Beals [1, Lemma 4] we can show that $Y$ is identified as $\bigcup_{h>0} Y(h)$, and complete the proof of Theorem 2. 
Lemma 8. There is a positive number $q$ such that

$$
\begin{array}{ll}
U(s) X \subset Y(h) & \text { for } 0<h<q s ; \\
Y(h) \subset U(s) X & \text { for } 0<s<q h,
\end{array}
$$

where the inclusion mappings are continuous.

\section{REFERENCES}

1. R. Beals, Semigroups and abstract Gevrey spaces, J. Funct. Anal. 10 (1972), 300-308.

2. __ Hyperbolic equations and systems with multiple characteristics, Arch. Rational Mech. Anal. 48 (1972), 123-152.

3. G. Da Prato and E. Giusti, Una caratterizzazione dei generatori di funzioni coseno astratte, Boll. Un. Mat. Ital. 22 (1967), 357-362.

4. H. O. Fattorini, Ordinary differential equations in linear topological spaces. I, J. Differential Equations 5 (1968), 72-105.

5. __ Ordinary differential equations in linear topological spaces. II, J. Differential Equations 6 (1969), 50-70.

6. J. A. Goldstein, Semigroups and second-order differential equations, J. Funct. Anal. 4 (1969), 50-70.

7. A. Guzman, Growth properties of semigroups generated by fractional powers of certain linear operators, J. Funct. Anal. 23 (1976), 331-352.

8. J. Kisyński, On cosine operator functions and one-parameter groups of operators, Studia Math. 44 (1972), 93-105.

9. M. Sova, Cosine operator functions, Rozprawy Mat. 49 (1966), 1-47.

10. M. Watanabe, Cosine families of operators and applications, Lecture Notes in Math., vol. 1223, Springer-Verlag, Berlin, Heidelberg and New York, 1986, pp. 278-292.

11. A. Yagi, Applications of the purely imaginary powers of operators in Hilbert spaces, J. Funct. Anal. 73 (1987), 216-231.

12. K. Yosida, An operator-theoretical integration of the wave equation, J. Math. Soc. Japan 8 (1956), 79-92.

Faculty of General Education, Nitgata University, IKarashi 2-8050, Nitgata, 950-21 JAPAN 